STRUCTURAL BIOLOGY

ISSN 2059-7983

\title{
Structures of the transcriptional regulator BgaR, a lactose sensor
}

\author{
Janet Newman, ${ }^{\mathrm{a}}$ Karine Caron, ${ }^{\mathrm{b}}$ Tom Nebl ${ }^{\mathrm{a}}$ and Thomas S. Peat ${ }^{\mathrm{a} *}$
}

a Biomedical Program, CSIRO, 343 Royal Parade, Parkville, VIC 3052, Australia, and ${ }^{\mathbf{b}}$ Health and Biosecurity, CSIRO, Clunies Ross Street, Black Mountain, ACT 2601, Australia. *Correspondence e-mail: tom.peat@csiro.au

Received 28 March 2019

Accepted 6 June 2019

Edited by C. S. Bond, University of Western Australia, Crawley, Australia

Keywords: transcriptional regulators; BgaR; Clostridium perfringens; lactose sensors; SAD phasing.

PDB references: BgaR, mercury derivative, 6 nwh; complex with lactose, space group $P 22_{1} 2_{1}, 6$ nwj; space group $P 2_{1}, 6 n \times 3$; complex with lactulose, 6nwm; partial apo, 6nwo

Supporting information: this article has supporting information at journals.iucr.org/d

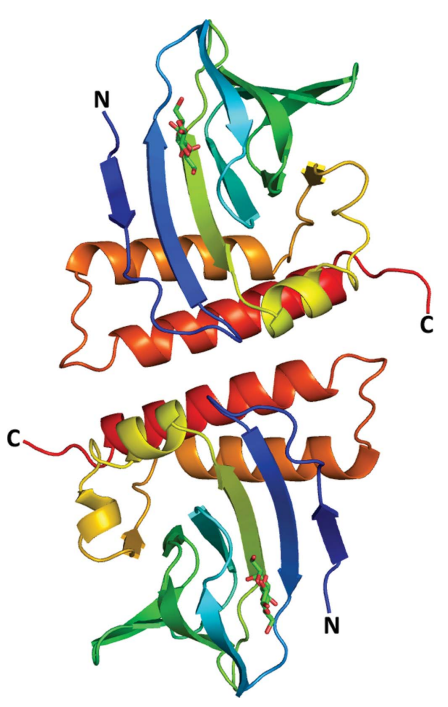

OPEN $\odot$ ACCESS
The structure of BgaR, a transcriptional regulator of the lactose operon in Clostridium perfringens, has been solved by SAD phasing using a mercury derivative. BgaR is an exquisite sensor of lactose, with a binding affinity in the low-micromolar range. This sensor and regulator has been captured bound to lactose and to lactulose as well as in a nominal apo form, and was compared with $\mathrm{AraC}$, another saccharide-binding transcriptional regulator. It is shown that the saccharides bind in the N-terminal region of a jelly-roll fold, but that part of the saccharide is exposed to bulk solvent. This differs from the classical AraC saccharide-binding site, which is mostly sequestered from the bulk solvent. The structures of BgaR bound to lactose and to lactulose highlight how specific and nonspecific interactions lead to a higher binding affinity of BgaR for lactose compared with lactulose. Moreover, solving multiple structures of BgaR in different space groups, both bound to saccharides and unbound, verified that the dimer interface along a $\mathrm{C}$-terminal helix is similar to the dimer interface observed in AraC.

\section{Introduction}

Living organisms have evolved a broad range of sensing systems for the detection of small molecules, such as metabolites and nutrients, and also of temperature and $\mathrm{pH}$ variations. Transcriptional regulators (TRs) play a major role in sensing small molecules and are a broad family of proteins that control cell development, cell differentiation and cell growth through the regulation of gene expression, and these proteins are found throughout all biological kingdoms. The mechanism by which TRs regulate gene transcription varies amongst this family of proteins. Often, a small molecule such as a metabolite or a nutrient binds to the effector-binding domain, leading to a conformation change that alters the interaction of the DNA-binding domain with the target DNA, ultimately affecting the efficiency of gene transcription. TRs have been divided into a broad range of families based on structural and binding similarities. GntR-family members (named after the gluconate operon repressor in Bacillus subtilis) are characterized by having a somewhat conserved DNA-binding domain linked to a diverse globular regulatory effectorbinding domain. The structural divergence amongst the TRs of the GntR superfamily gives rise to six subfamilies: four main subfamilies (FadR, HutC, MocR and YtrA) and two minor subfamilies (AraC and PlmA) (Jain, 2015; Rigali et al., 2002).

To date, only a few AraC-family members have been structurally characterized. X-ray structures are available for the MarA (Rhee et al., 1998), Rob (Kwon et al., 2000), ToxT (Lowden et al., 2010) and AraC TRs. For the AraC regulator the structures of the regulatory (Soisson et al., 1997) and DNA-binding (Rodgers \& Schleif, 2009) domains were solved 
separately. TRs of the AraC subfamily are mainly involved in the regulation of carbon metabolism (AraC), stress response (SoxS, Rob and MarA) and the regulation of virulence gene expression [for example Rns from enterotoxigenic Escherichia coli (Munson \& Scott, 1999), BfpT from enteropathogenic E. coli (Tobe et al., 1996) and ExsA from Pseudomonas aeruginosa (Hovey \& Frank, 1995)].

BgaR from Clostridium perfringens is a putative AraCfamily TR with lactose (Hartman et al., 2011; Caron \& Trowell, 2018) and, less efficiently, lactulose as effectors (Caron \& Trowell, 2018). Lactose is a commonly found disaccharide which consists of galactose and glucose moieties joined by a $\beta-1 \rightarrow 4$ glycosidic link. Lactulose is a synthetic disaccharide that is formed from lactose by heat, and consists of linked galactose and fructose monosaccharides. Lactulose is an important product that is cited on the World Health Organization's list of Essential medicines and health products (https://www.who.int/ medicines/news/2017/20th_essential_med-list/en/) and which has been used as a heat-treatment indicator in milk for over half a century (Adachi \& Patton, 1961). Little is known about the BgaR protein or its interaction with its known effectors. It is of interest to uncover the structure of BgaR and to understand the ligand-binding mechanism of saccharides to this regulatory domain, which would allow its comparison with $\mathrm{AraC}$ and a more extensive characterization of this lactose biosensor. In turn, this would expand our understanding of this family of carbon-metabolism regulators and our understanding of the differences in binding affinity between these regulatory proteins. This would then allow the engineering of the protein to be a sensor for lactose in products that are to be labelled 'lactose-free'. This paper describes the purification, crystallization and structure determination by SAD phasing of the regulatory domain of BgaR bound to lactose and to lactulose, as well as a nominal apo form of the protein, in several different space groups. We then compare this new structure with the 'classical' TR regulatory binding domain of AraC.

\section{Materials and methods}

\subsection{Cloning of BgaR ${ }_{1-170}$-thrombin-His 6 into pRSET}

The DNA sequence encoding the regulatory domain of BgaR (C. perfringens strain 13) was codon-optimized for expression in $E$. coli and synthesized commercially (GenScript, USA). A fragment, $\mathrm{BgaR}_{1-170}$-thrombin-His 6 , was amplified from the pUC-BgaR vector using the primers shown in Table 1. The BgaR ${ }_{1-170}$-thrombin-His ${ }_{6}$ fragment was cloned into the pRSET vector (BioLabs, Australia) using the NdeI and HindIII endonucleases. The integrity and orientation of the clone were verified by sequencing.

\subsection{Expression and purification of $\mathrm{BgaR}_{1-170}$}

BgaR $_{1-170}$-thrombin-His 6 was expressed in E. coli BL21 (DE3) cells (New England Biolabs). $50 \mathrm{ml}$ lysogeny broth (LB) supplemented with $2 \%(\mathrm{~m} / \mathrm{v})$ glucose and $100 \mu \mathrm{g} \mathrm{ml}{ }^{-1}$ ampicillin was inoculated with a single colony and cultured at
Table 1

Macromolecule-production information.

\begin{tabular}{ll}
\hline Source organism & C. perfringens strain 13 \\
DNA source & Synthetic \\
Forward primer & AAAAAACATATGCAGATTCTGTGGAAA \\
Reverse primer & TATATAAAGCTTTTAATGATGATGATGATG \\
& ATGGCTGCCGCGCGGCACCAGATGCAGT \\
& TCTTTATC \\
Cloning vector & pUC \\
Expression vector & pRSET \\
Expression host & E. coli BL21 (DE3) \\
Complete amino-acid sequence & MQILWKKYVKENFEMNVDEGIEQGIPGLG \\
\multicolumn{1}{c}{ of the construct produced } & YNYEVLKNAVIHYVTKGYGTFKFNGKVY \\
& NLKQGDIFILLKGMQVEYVASIDDPWEY \\
& YWIGFSGSNANEYLNRTSITNSCVANCE \\
& ENSKIPQIILNMCEISKTYNPSRSDDIL \\
& LLKELYSLLYALIEEFPKPEYKDKELH \\
& LVPRGSHHHHH \\
\hline
\end{tabular}

$37^{\circ} \mathrm{C}$ and $200 \mathrm{rev} \min ^{-1}$ until it reached an $\mathrm{Abs}_{600 \mathrm{~nm}}$ of 0.8 . The starter culture was used to inoculate $250 \mathrm{ml}$ LB supplemented with $100 \mu \mathrm{g} \mathrm{ml}^{-1}$ ampicillin at an $\mathrm{Abs}_{600 \mathrm{~nm}}$ of 0.05 , which was incubated at $28^{\circ} \mathrm{C}$ and $200 \mathrm{rev} \mathrm{min}^{-1}$ for $24 \mathrm{~h}$. No addition of IPTG or any other induction compound was needed. The cells were harvested and were lysed using a homogenizer (Microfluidics M-110P, $137 \mathrm{MPa}$ ). The $\mathrm{His}_{6^{-}}$ tagged protein was purified using TALON Superflow Metal Affinity Resin (Takara Clontech, Australia) according to the manufacturer's instructions [the crude lysate was applied onto the resin, washed with equilibration buffer $(50 \mathrm{~m} M$ sodium phosphate, $300 \mathrm{~m} M$ sodium chloride $\mathrm{pH}$ 7.0), washed with $10 \mathrm{~m} M$ imidazole in equilibration buffer ( $\mathrm{pH} 7.0)$ and eluted with $150 \mathrm{~m} M$ imidazole in equilibration buffer ( $\mathrm{pH}$ 7.4)]. The eluted protein was dialysed against $50 \mathrm{~m} M$ bis-Tris $\mathrm{pH} 6.5$, $50 \mathrm{~m} M$ sodium chloride buffer using a D-Tube Dialyzer (Merck; 6-8 kDa molecular-weight cutoff). The purity was assessed by SDS-PAGE (Supplementary Fig. S1) with an approximate size of $20 \mathrm{kDa}$. Protein concentrations were estimated by measurement of $\mathrm{Abs}_{280 \mathrm{~nm}}$. The protein was flashfrozen in $100 \mu \mathrm{l}$ aliquots in thin-walled PCR tubes and stored at $-80^{\circ} \mathrm{C}$.

\subsection{Crystallization}

All crystallization trials were performed at the $\mathrm{C} 3$ centre (http://crystal.csiro.au) in SD-2 ('MRC') sitting-drop plates using a Phoenix (Art Robbins Instruments, USA) or a Mosquito (TTP Labtech, UK) robot. Experiments were stored and imaged using an RI1000 incubator system (Formulatrix, USA). Initial experiments ( $150 \mathrm{nl}$ protein solution plus $150 \mathrm{nl}$ reservoir solution equilibrated against $50 \mu \mathrm{l}$ reservoir solution) were set up with the protein at $5 \mathrm{mg} \mathrm{ml}^{-1}$ in $50 \mathrm{mM}$ sodium chloride, $50 \mathrm{~m} M$ bis-Tris pH 6.5 with $1 \mathrm{~m} M$ lactulose with or without thrombin $(100 \mu$ l protein solution added to $10 \mu \mathrm{g}$ thrombin). Screens tested included Shotgun (Fazio et al., 2014) at both 20 and $8^{\circ} \mathrm{C}$, as well as PACT and PSgradient both at $20^{\circ} \mathrm{C}$. Detailed descriptions of these screens are available from the C6 website (http://c6.csiro.au; Newman et al., 2010). Spherulites from the (thrombin) trials were optimized using a combination of fine screening, microseeding and the substitution of either trypsin or chymotrypsin for the thrombin 
Table 2

Crystallization.

\begin{tabular}{ll}
\hline Method & Vapour diffusion, sitting drop \\
Plate type & SD-2 \\
Temperature (K) & 293 \\
Protein concentration $\left(\mathrm{mg} \mathrm{ml}^{-1}\right)$ & 5 \\
Buffer composition of protein solution & $50 \mathrm{~m} M$ bis-Tris pH $6.5,50 \mathrm{~m} M \mathrm{NaCl}$ \\
Composition of reservoir solution & See text \\
Volume and ratio of drop & $200 \mathrm{nl}+200 \mathrm{nl}$ drops \\
Volume of reservoir $(\mu \mathrm{l})$ & 50 \\
\hline
\end{tabular}

protease. After refinement, crystals grew over the course of several days in many different conditions. Trials were set up with protein plus $1 \mathrm{~m} M$ lactose, protein plus $1 \mathrm{~m} M$ lactulose or protein with no added sugar.

Three different mercury compounds (mercuric cyanide, mersolyl acid and $p$-hydroxymercuribenzoic acid) were tested as phasing vehicles; in each case a $2 \mathrm{mM}$ solution of the heavy atom in the reservoir mixture was added to the crystal and left to soak for 1-3 days. Crystallization information is given in Table 2.

\subsection{Crystallography}

Before harvesting, crystals grown in PEG 3350 were cryoprotected by adding glycerol to the reservoir to give a final concentration of $20 \%$ and then layering $1 \mu \mathrm{l}$ of this solution over the crystal-containing drops. Crystals harvested from sodium malonate-containing conditions were cryoprotected by the addition of $3.4 \mathrm{M}$ sodium malonate to the reservoir to a final concentration of $1.7 \mathrm{M}$ and layering $1 \mu \mathrm{l}$ of the modified reservoir solution over the drops before fishing out the crystals. Data were collected on the microfocus (MX2) beamline at the Australian Synchrotron using an EIGER 16M detector. For each crystal, a data set $\left(360^{\circ}\right)$ was collected over 36 s. For the mercury derivative, the energy of the X-ray beam was set to $12300 \mathrm{eV}$ ( $1.008 \AA$; i.e. close to the $\mathrm{Hg}$ peak) for SAD phasing and two data sets were collected from the same crystal, moving down the crystal for the second data set (see Table 3 for data-collection statistics). The monoclinic crystal (PDB entry $6 \mathrm{n} \times 3$ ) had been soaked with sodium iodide and a data set was obtained with the wavelength set to $1.45865 \AA$ $(8500 \mathrm{eV})$ to obtain phase information from a possible iodine derivative (no anomalous signal was observed). The beam energy was set to $13000 \mathrm{eV}$ for the other data sets. The data were indexed using $X D S$ (Kabsch, 2010) and scaled using AIMLESS (Evans \& Murshudov, 2013); CRANK2 (Skubák \& Pannu, 2013) was used to obtain the $\mathrm{Hg}$ sites, the initial phasing and the initial structure. The structure was rebuilt manually using Coot (Emsley et al., 2010) and refined using REFMAC (Murshudov et al., 2011). All subsequent structures were phased using Phaser (McCoy et al., 2007) using PDB entry 6nwh as the starting model, manually rebuilt using Coot and refined using REFMAC.

Monoclinic crystals (space groups $I 2$ and $P 2_{1}$ ) were harvested from drops where the reservoir contained 20-22\% PEG 3350, 200-220 mM MgCl 2 with either $100 \mathrm{~m} M$ bis-Tris $\mathrm{pH} 5.7$ or no added buffer. Orthorhombic crystals (space group $P 2_{1} 2_{1} 2$ ) were harvested from drops where the reservoir contained $1.25-1.33 M$ sodium malonate in a variety of buffers [100 $\mathrm{m} M$ citrate $\mathrm{pH} 5.5$ (PDB entry $6 \mathrm{nwh}$ ), $100 \mathrm{~m} M$ HEPES $\mathrm{pH} 7.5$ (PDB entry 6nwm) or $100 \mathrm{~m} M$ glycylglycine $\mathrm{pH} 8.2$ (PDB entry 6nwj)]. Crystals grown in sodium malonate and citrate buffer and soaked with mercuric cyanide were used to collect the derivative data set used in phasing.

\subsection{Mass spectrometry}

Samples of $\mathrm{BgaR}_{1-170}$-thrombin-His ${ }_{6}$ treated with thrombin, trypsin or chymotrypsin, as well as the untreated protein, were analysed by mass spectrometry. For accurate mass determination, protein samples were spiked with formic acid (FA) to a final concentration of $0.1 \%(v / v)$ and separated by reversephase liquid chromatography on an UltiMate 3000 RSLCnano system (Thermo Fisher Scientific) fitted with a $50 \times 4.6 \mathrm{~mm}$, $5 \mu \mathrm{m}$ particle-size, $300 \AA \AA$ A pore-size PLRP-S column (Agilent). Proteins were eluted at a flow rate of $250 \mu \mathrm{min}^{-1}$ by applying a linear 30 min gradient from 0 to $80 \%$ solvent $B$ [mobile phase $A, 0.1 \%(v / v) \mathrm{FA}$; mobile phase $B, 90 \%(v / v)$ acetonitrile/ $0.1 \%(v / v)$ FA] and were ionized using an Apollo II electrospray ion source coupled to a micrOTOF-Q II mass spectrometer (Bruker). The native protein appeared as multiple species in the analysis, corresponding to various $\mathrm{C}$-terminal truncations of the full-length construct (see Supplementary Fig. S2). The thrombin-treated sample was very clean by comparison, showing a single peak corresponding to cleavage after the arginine in the thrombin cleavage site. Both the trypsin-treated and the chymotrypsin-treated samples gave results consistent with cleavage close to the $\mathrm{C}$-terminus: after Lys165 and Tyr164, respectively. Chymotrypsin treatment gave two equal-sized peaks: one consistent with cleavage after Tyr164 and one that was 1091 mass units smaller (data not shown).

Native mass spectra were acquired using a maXis II mass spectrometer (Bruker Daltonics) equipped with a standard ESI source in positive-ion mode. Protein samples were exchanged into $200 \mathrm{mM}$ ammonium acetate buffer using Zeba Spin desalting columns (Thermo Fisher Scientific), and native MS was performed at a flow rate of $3 \mu \mathrm{min}^{-1}$ using a direct infusion mode. To guarantee the ionization effect of the sample solution, the capillary voltage and the end-plate offset were maintained at 3200 and $-500 \mathrm{~V}$, respectively. The temperature and the flow rate of the drying gas were maintained at $120^{\circ} \mathrm{C}$ and $31 \mathrm{~min}^{-1}$, respectively. The nebulizer gas pressure was 0.4 bar. To achieve a favourable desolvation effect and increase the sensitivity, the isCID and collision energies were set to 100 and $7 \mathrm{eV}$, respectively. Quadrupole frequencies, RF amplitudes and transfer times were adjusted to achieve the best ion-transmission efficiency. Native MS data were acquired at $1 \mathrm{~Hz}$ and were processed using parsimonious charge deconvolution (Bern et al., 2018).

\subsection{Dimerization of the regulatory domain}

The protein was diluted to $0.2 \mathrm{mg} \mathrm{ml}^{-1}$ in $50 \mathrm{~m} M$ bis-Tris $\mathrm{pH} 6.5,50 \mathrm{~m} M \mathrm{NaCl}$ for dynamic light-scattering experiments. A Wyatt DynaPro DLS plate reader was used for data 
Table 3

Data collection and refinement.

Values in parentheses are for the outer shell.

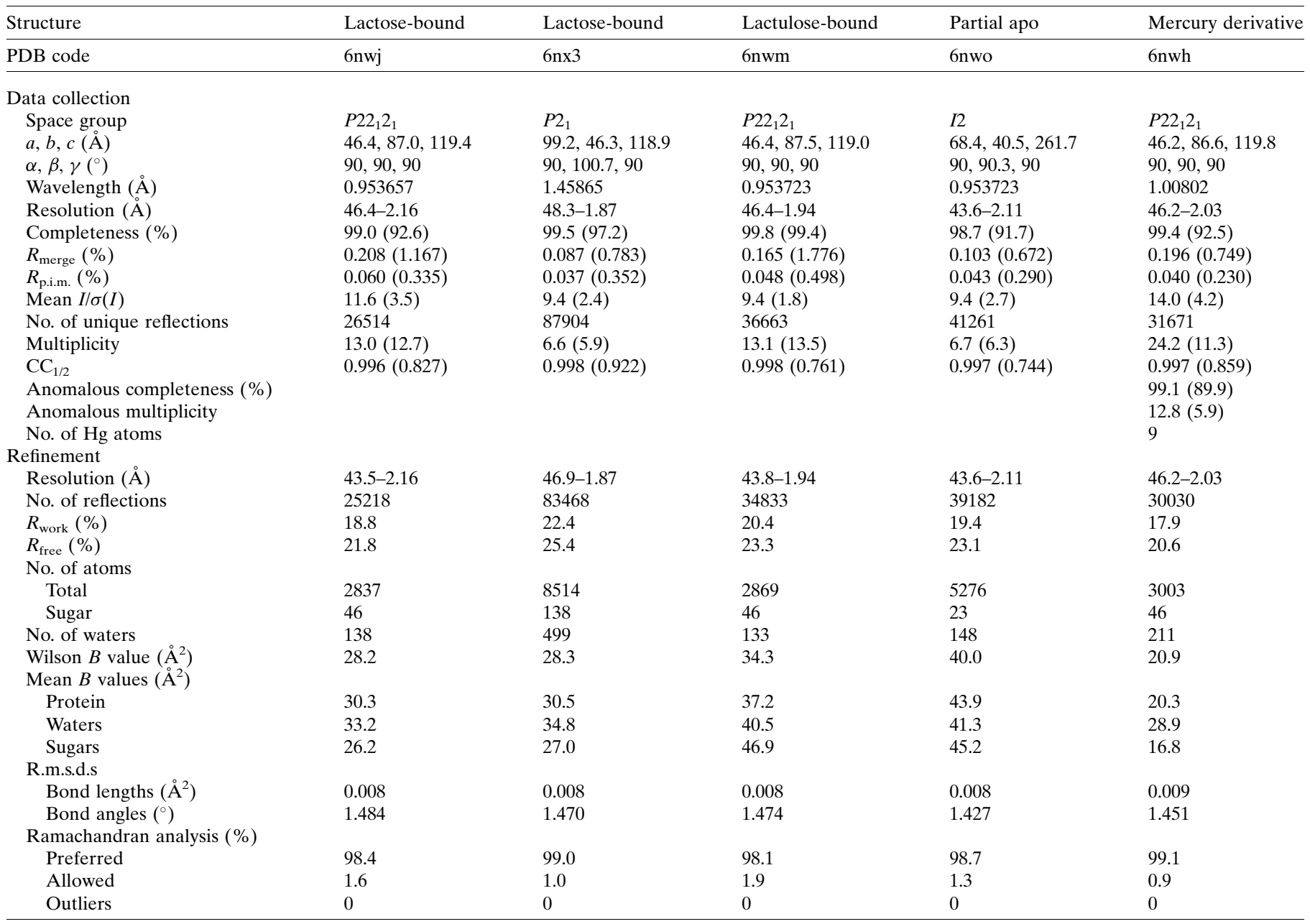

collection, which was performed at $25^{\circ} \mathrm{C}$ with $20 \times 5$ s readings. The average size of the particles was $2.6-2.7 \mathrm{~nm}$, giving an estimated molecular weight of $32-35 \mathrm{kDa}$ (data not shown). Additionally, $400 \mu \mathrm{l}$ of protein solution (about $2 \mathrm{mg}$ ) was subjected to size-exclusion chromatography using a Superdex 200 column (GE Healthcare) in phosphate-buffered saline solution $(9 \mathrm{~m} M$ sodium potassium phosphate $\mathrm{pH} 7.4$ with $137 \mathrm{mM} \mathrm{NaCl}$ and $3 \mathrm{mM} \mathrm{KCl}$ ) with the protein eluting at $16.5 \mathrm{ml}$, close to the standard chicken ovalbumin (44 kDa), which elutes at $15.9 \mathrm{ml}$ (see Supplementary Fig. S3). Using linear interpolation, this gives a molecular weight of 36$37 \mathrm{kDa}$ for the BgaR peak.

\section{Results and discussion}

The full-length BgaR protein yielded crystals, but these did not diffract beyond 7-8 $\AA$ resolution. The shorter regulatory (ligand-binding) domain construct $\left(\mathrm{BgaR}_{1-170}\right.$-thrombin-His $\left.{ }_{6}\right)$ only gave crystals when treated with a protease, which resulted in the removal of the $\mathrm{C}$-terminal tag and five or six residues from the C-terminus. Thrombin, chymotrypsin and trypsin were all tried in in situ proteolysis experiments. Thrombin treatment, which cleaved off only the tag, gave drops that contained promising spherulites but that would occasionally produce single crystals with seeding. Trypsin and chymotrypsin both removed the tag and a small number of C-terminal residues and reliably gave single crystals, although trypsin treatment generally produced better diffracting crystals, probably as the in situ trypsin treatment produced essentially a single product, whereas chymotrypsin treatment gave two major products according to the mass-spectrometric analysis. Mass spectrometry showed a mass of 20312 Da for monomeric BgaR under denaturing conditions and also showed that the protein flew as a 40624 Da dimer under native conditions (see Supplementary Fig. S2). The most complete structures showed density from residue 1 through to residue 162, and no density was seen for residues 163-166.

The dimer seems to be the prevalent form in solution based on size-exclusion chromatography (see Supplementary Fig. S3), dynamic light scattering (see Section 2) and crystallography (see below), as well as native-state mass-spectrometric data (Supplementary Fig. S2). The BgaR dimer interface is dominated by a long C-terminal helix which forms the majority of the interactions, having both hydrophilic and 
hydrophobic components (Fig. 1). The hydrophobic residues include Ile141, Leu142, Leu144, Tyr99, Tyr148 and Tyr152,
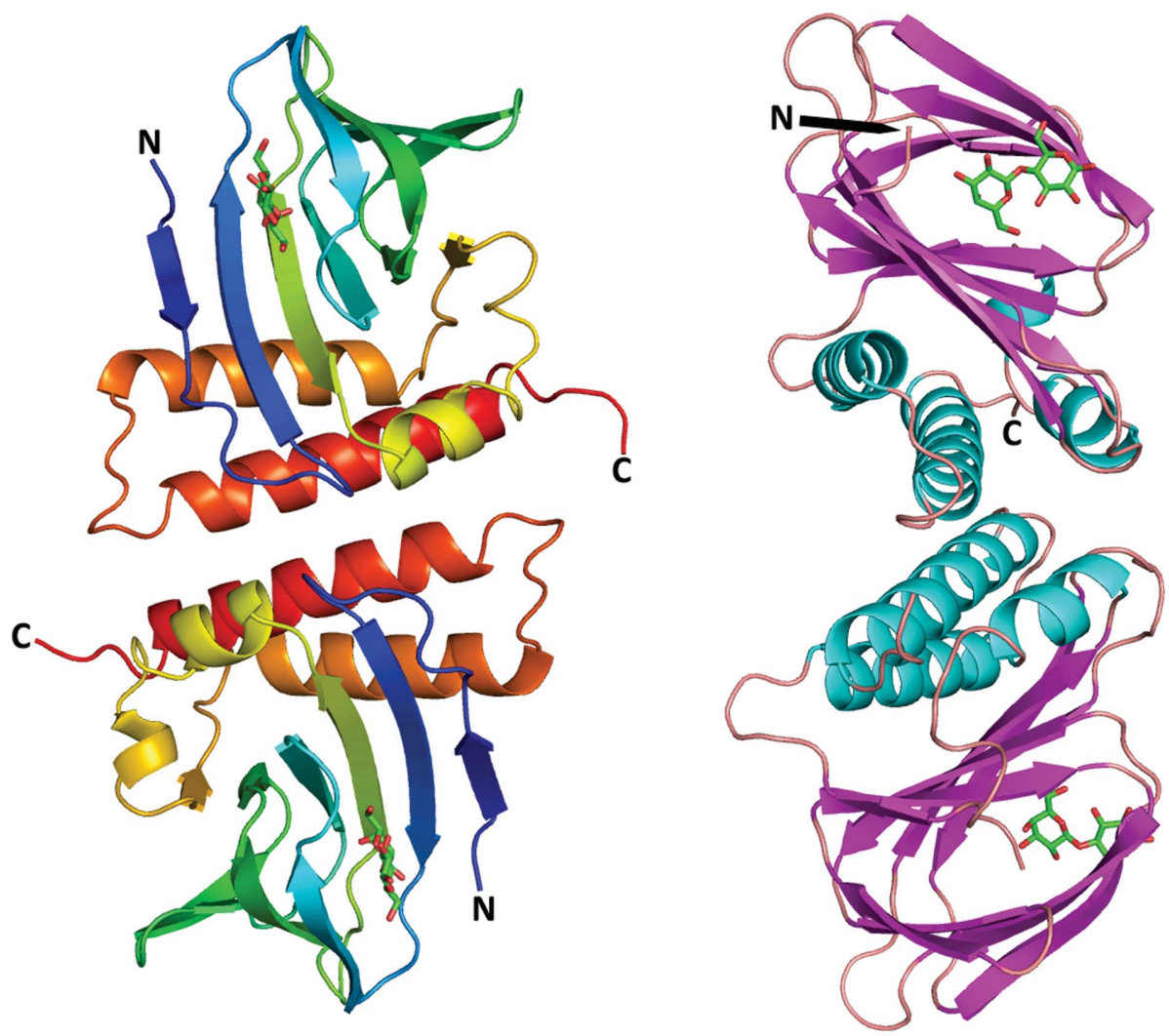

Figure 1

The BgaR dimer bound to lactose. The image on the left depicts the dimer as a cartoon coloured as a Jones' rainbow from the $\mathrm{N}$-terminus to the $\mathrm{C}$-terminus. Lactose is shown as a stick model with $\mathrm{C}$ atoms in green. The image on the right is an approximate $90^{\circ}$ rotation and the colours have been changed to highlight the secondary structure ( $\beta$-strands in magenta and $\alpha$-helices in cyan). Lactose is shown as a stick model with $\mathrm{C}$ atoms in green. The dimer is held together by the long $\mathrm{C}$-terminal helices and the saccharide-binding site is between the $\beta$-sheets of the jelly-roll fold. whereas the hydrophilic residues include Glu14, Asn12, Asn95, Arg102 and Asp140. Lys145 also sits at the interface, but it is the aliphatic chain of this residue which is presented to its neighbour instead of the amino head, which forms a same-chain bond with Glu146. Analysis using PISA (Krissinel \& Henrick, 2007) suggests that the protein forms a dimer in solution with almost $20 \%$ of the surface area buried in the interface $\left(2940 \AA^{2}\right.$ buried out of a total of $15380 \AA^{2}$ ). This dimer is also seen in the two monoclinic crystal forms and in the orthorhombic crystals over a broad range of $\mathrm{pH}$ values (5.5-8.2).

The most similar protein in the PDB is the regulatory domain of AraC (Soisson et al., 1997), which shares 17\% sequence identity with that of $\mathrm{BgaR}$, and the best AraC/BgaR structure alignment gives an r.m.s.d. of $2.2 \AA$ over 133 residues (out of 161/162). The available AraC structures did not provide useful solutions in molecular-replacement trials, even though the overall fold is quite similar: a jelly roll of eight (or nine) $\beta$-strands with $\mathrm{C}$-terminal helices (Fig. 1), where the C-terminal helix forms the dimer interface. The crystal structures of BgaR with added saccharides gave models in which there was clear density from residue 1 through to residue 161 or 162 . Although extensive hydrogen-bond networks and stacking interactions are made with the
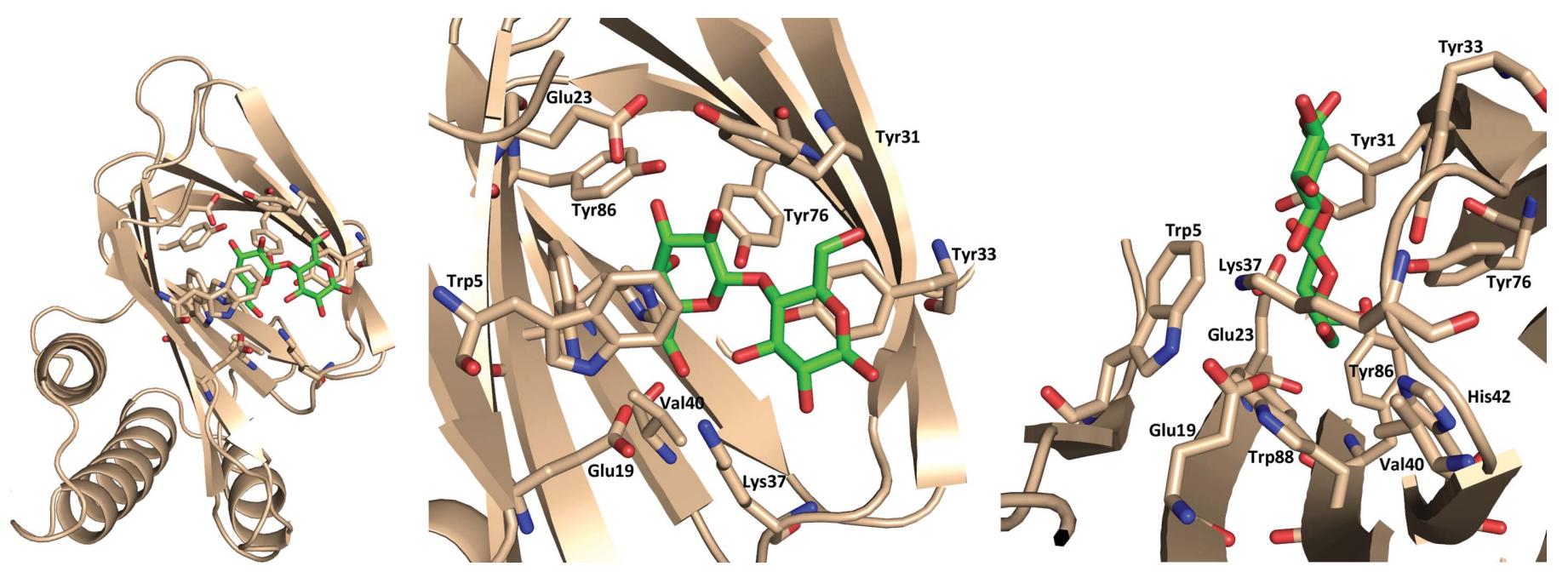

Figure 2

Monomer of BgaR bound to lactose: detailed view. The image on the left shows lactose in a stick representation with green $\mathrm{C}$ atoms within a monomer of $\mathrm{BgaR}$. The residues involved in binding to the lactose are also shown in stick representation. The middle image is an enlarged view highlighting the individual residues (labelled) and the image on the right is rotated to show that although one half of the saccharide (galactose) is deeply engaged in the binding site, the other half (the glucose ring) is mostly exposed to solvent. 
saccharide (Fig. 2), part of the lactose (or lactulose) moiety is open to bulk solvent, with only the common galactose ring being sequestered significantly for both sugars.

The saccharide-free crystals showed some disorder in the first ten residues, and these residues are not modelled in two of the four protomers. Although no lactose/lactulose was added to the protein for the apo crystals, there is some density in one of the binding sites (four protomers are seen in the asymmetric unit). Native mass spectrometry detected a +342.2 Da mass addition consistent with lactose binding to the $\mathrm{BgaR}$ dimer (Supplementary Fig. S2), and we have therefore modelled this density as lactose. In this case the galactose ring has clear density, with the glucose ring having clear but weaker density. No obvious saccharide density is seen in two of the other four
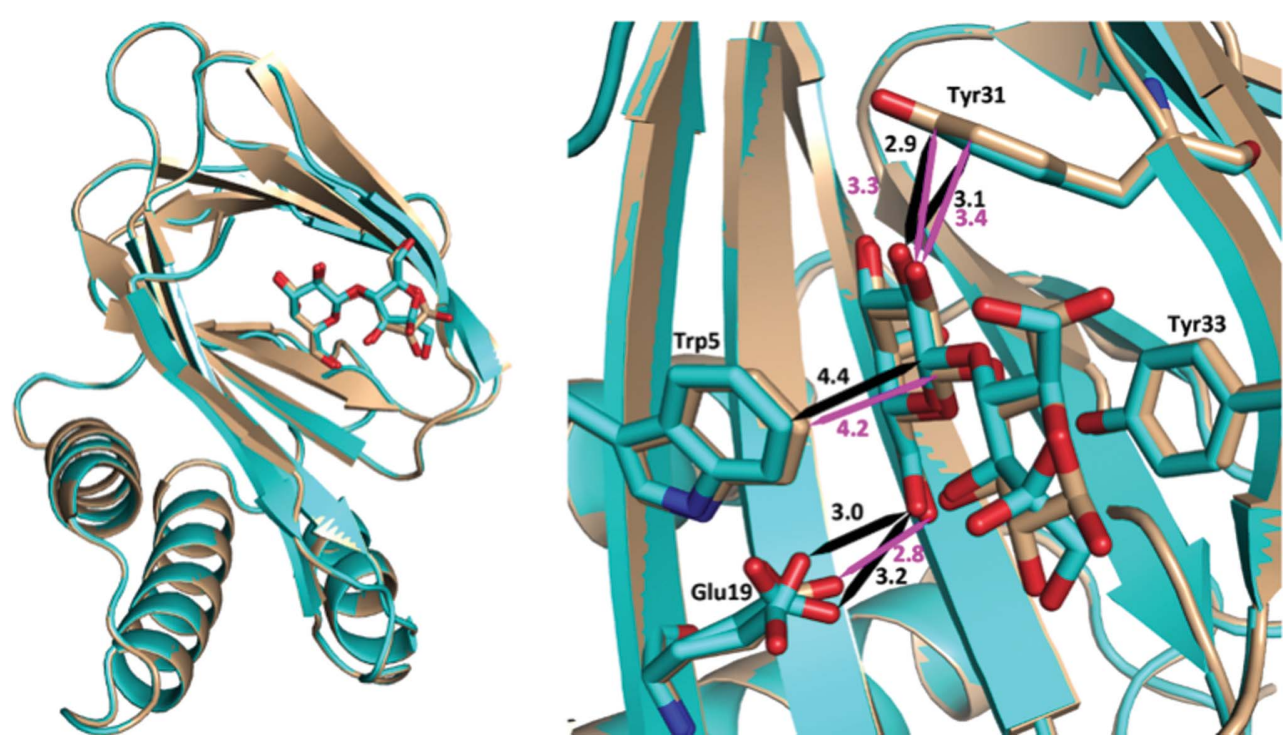

Figure 3

Lactose and lactulose binding in BgaR. The image on the left shows a superposition of the monomers of the lactose-bound and lactulose-bound BgaR structures, showing that the saccharides bind in the same pocket in a very similar way; the lactulose-bound structure is shown in cyan and the lactose-bound structure is in wheat. The image on the right is an enlarged perspective with distances shown as black arrows/type for the lactulose-bound structure and in magenta for the lactose-bound structure. A slight shift in the galactose moiety towards the viewer is seen in the lactulose-bound structure. Slight rearrangements in the glucose/ fructose ring cause a galactose hydroxyl to move into close proximity to the aromatic C atoms of Tyr31, cause another hydroxyl to make a weaker hydrogen bond to Glu19 and reduce the stacking interactions of the saccharide between Trp5 and Tyr33.

binding sites and some weak density for a glycerol molecule occupies one side of the binding site in the last protomer. There is some unmodelled density in one of the two 'empty' saccharidebinding sites of the apo structure. Comparing the saccharide-free structure with that with lactose bound shows an overall r.m.s.d. of 0.3-0.4 $\AA$ for $\mathrm{C}^{\alpha}$ atoms (151 residues aligned), with the largest changes in the binding site being a different rotomer position for Trp88 and shifts of $0.5-1.0 \AA$ in the $\mathrm{C}^{\alpha}$ positions of Tyr31 (depending on the protomer compared). As above, the first ten residues of the saccharide-free protomers are not modelled, including Trp5, which is also part of the binding site.

Comparing the structures of the lactose-bound, the lactulosebound and the partial apo structure and the different space groups allow us to more fully
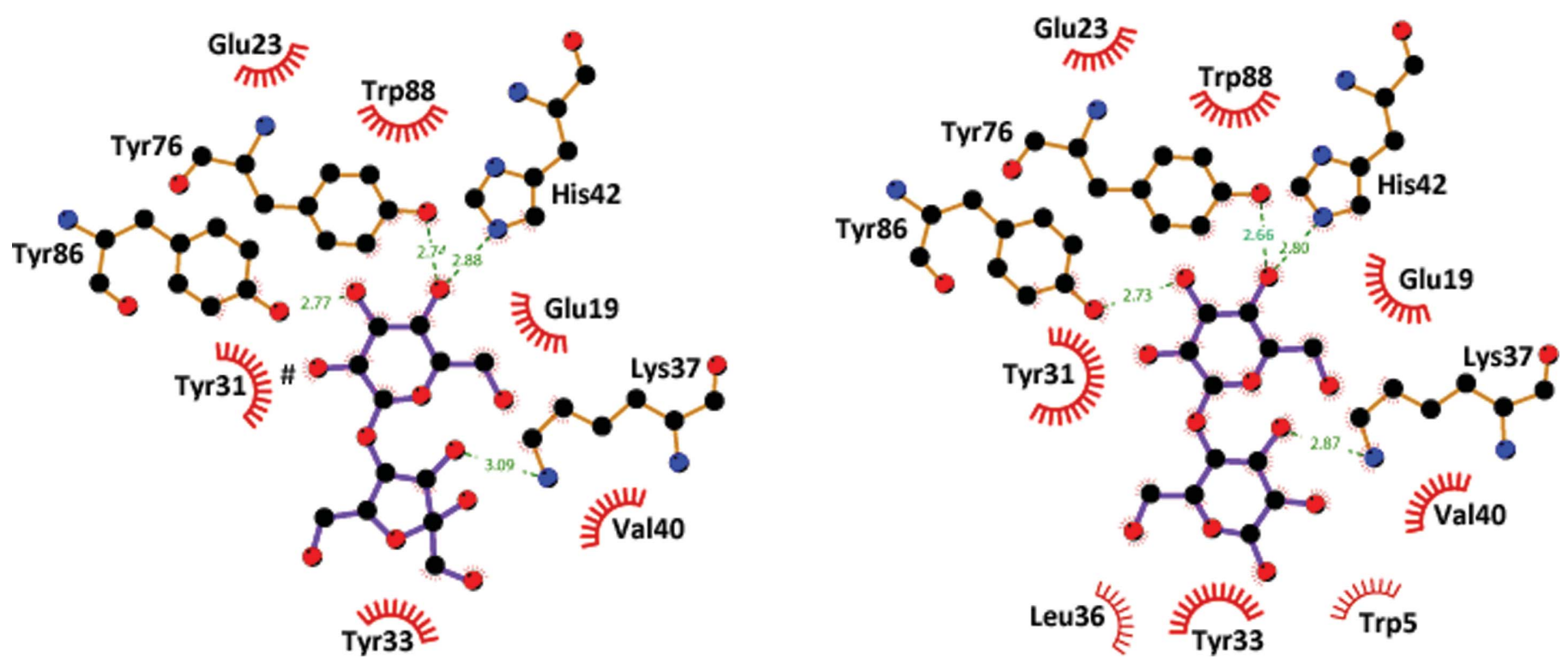

Figure 4

LigPlot+ representation of lactose and lactulose binding in BgaR. Lactulose binding is shown on the left and lactose binding is shown on the right. The \# mark in the lactulose-binding figure indicates a steric clash between one of the galactose $\mathrm{O}$ atoms and Tyr31. The hydrogen bonds made by lactose are shorter/stronger than those made by lactulose. This figure was produced using LigPlot+ (Laskowski \& Swindells, 2011). 
understand the saccharide-binding characteristics of this regulatory domain. From previous work, we know that lactose binds to the BgaR protein about 200 times more tightly than lactulose, glucose or galactose, with lactulose being the next best binder (Caron \& Trowell, 2018). BgaR binds lactose and lactulose through the same mechanism, and the same amino acids are involved (Figs. 3 and 4). Although many of the hydrogen bonds are shared between the lactose-bound and lactulose-bound structures, on average the bonds are slightly longer in the lactulose-bound structure. As seen in Fig. 4, two residues make contact with lactose (Leu36 and Trp5) which do not make contact with lactulose (according to LigPlot+ with default parameters; Laskowski \& Swindells, 2011). Additionally, there are some stacking interactions of the saccharide with the protein, and these also seem to be weaker (further apart) in the lactulose-bound structure than in the lactosebound structures (for example stacking with Tyr33). There are some close contacts with the aromatic $\mathrm{C}$ atoms of Tyr31 and
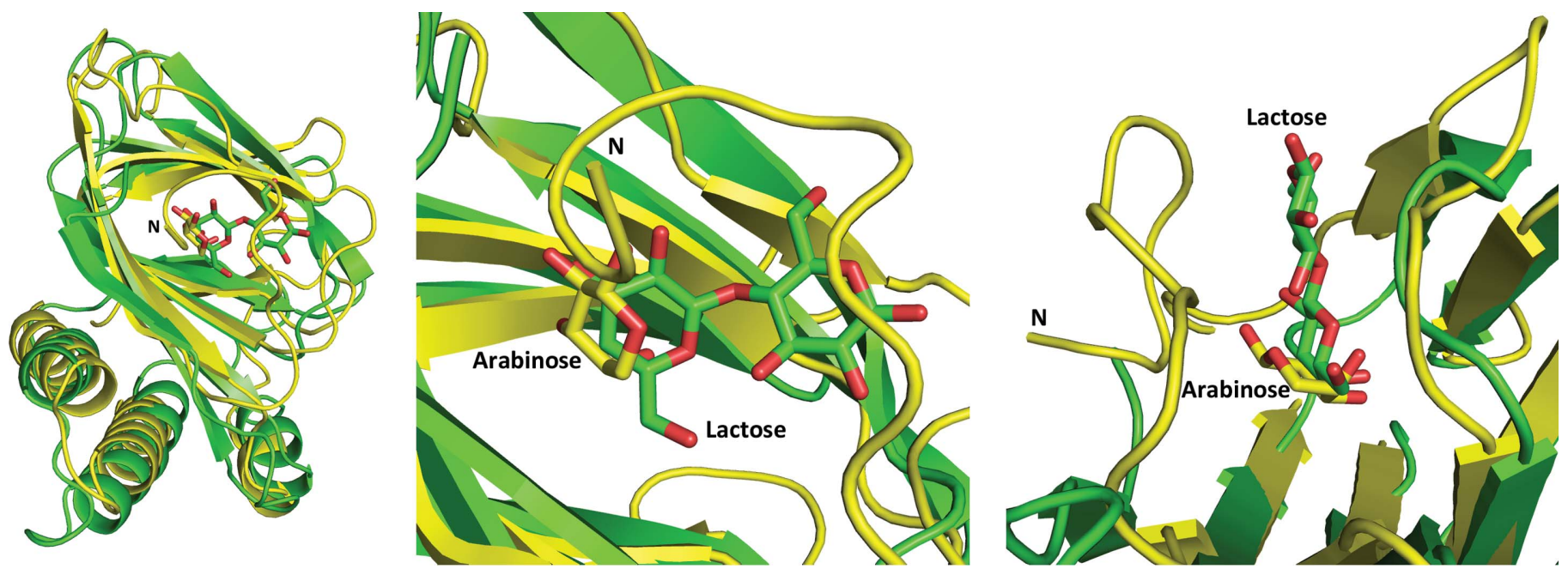

Figure 5

Comparison of lactose binding to BgaR and arabinose binding to AraC. The left image shows a comparison of the AraC structure with arabinose bound with the BgaR structure with lactose bound, showing that the overall folds are quite similar between the proteins and that the binding sites are both found between the $\beta$-sheets of the jelly roll; the AraC structure is in yellow and the BgaR structure is in green. The middle and right images are enlarged perspectives of the left image to show in detail how the arabinose sits at almost $90^{\circ}$ in the pocket compared with the lactose moiety. The N-terminus of AraC does not participate in the $\beta$-sheets formed but instead comes up to enclose the binding site for the saccharide, unlike the BgaR structure. The $\mathrm{N}$-terminus is labelled in each case.
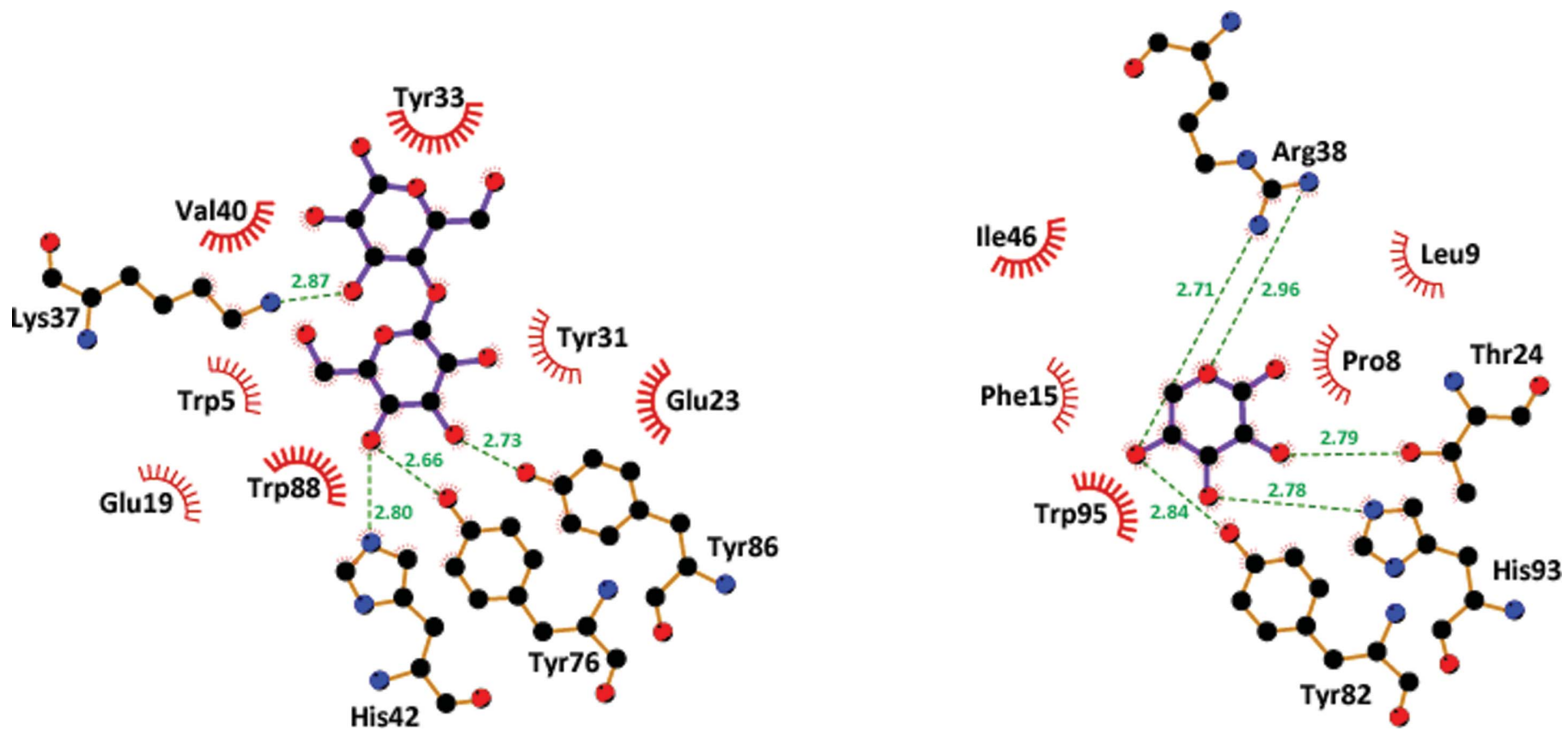

Figure 6

LigPlot+ representation of lactose binding to BgaR and arabinose binding to AraC. Lactose bound to BgaR is shown on the left and arabinose bound to AraC is shown on the right. In both cases, specific hydrogen bonds are made to the saccharides, as well as hydrophobic and stacking interactions. The residues at the $\mathrm{N}$-terminus are involved in binding; when no saccharide is present the binding site opens and the N-terminus becomes disordered. 
one of the hydroxyl $\mathrm{O}$ atoms on the galactose ring of lactulose which are not seen in the lactose-bound structure (2.9-3.1 $\AA$ instead of 3.2-3.4 $\AA$ ). This shift between the saccharides is subtle, changing hydrogen-bond distances, stacking distances and van der Waals interactions, which in total cause the lower binding affinity of lactulose compared with lactose.

The closest structures from the PDB are those of AraC. The overall fold is quite similar, with the major difference being at the N-terminus, where the AraC structure more effectively covers the saccharide-binding site with a large loop (Figs. 5 and 6). The N-terminus of the BgaR structure instead forms an extra strand in the $\beta$-sheet which passes through the middle of the protein, and this creates a more open saccharide-binding site. In both proteins there is a long C-terminal helix which forms the majority of the dimerization face, which has both hydrophilic and hydrophobic components. The saccharide is oriented differently in these two proteins. The arabinose is almost perpendicular to the galactose moiety of the lactose in the binding site of $\mathrm{AraC}$ when the two structures are superposed (Fig. 5). The arabinose is effectively sequestered from the bulk solvent by the AraC protein, whereas the lactose is only covered effectively on one side (the galactose moiety) and the glucose (or fructose in the case of lactulose) moiety is partially free to interact with the bulk solvent in the BgaR structures.

\section{Conclusion}

Transcriptional regulator-based biosensors have been developed and used broadly for decades owing to their inherent function in triggering a reporter signal in the presence of metabolites, nutrients and other solutes. However, whole-cell detection-system applications are limited to research settings owing to their slow response times and their requirements for skilled staff and specialized equipment. A few examples of modular TR-based biosensors have been reported in which the analyte-induced conformational change in the TR was captured by resonance energy transfer (RET). Caron and Trowell designed and built a lactose biosensor in which the BgaR protein was flanked with RET reporter proteins, namely GFP and the Renilla luciferase variant RLuc8. BgaR had the highest affinity for lactose $\left(\mathrm{EC}_{50}=12 \pm 1 \mu M\right)$ and a 200 -fold lower affinity for the analogue lactulose $\left(\mathrm{EC}_{50}=2.4 \pm\right.$ $0.2 \mathrm{mM}$ ) (Caron \& Trowell, 2018).

The crystal structures presented here show that lactose and lactulose bind in similar ways to the same residues in BgaR, but subtle differences in the binding modalities lead to different binding efficiencies. The higher affinity observed for lactose compared with lactulose may in part be owing to the short distances of Tyr31 to one of the hydroxyl atoms on the galactose ring of lactulose, but the stronger hydrogen bonds (e.g. Glu19), better stacking and additional residues making contact with lactose almost certainly contribute to stronger binding as well. We have shown using crystallography and native-state mass spectrometry that despite having little sequence similarity, the structure and dimer of BgaR are quite similar to those of AraC. Both proteins are transcriptional regulators that control the carbon metabolism of different saccharides and thus provide similar functions in their respective organisms. The current structure allows more rational engineering of this protein for future biosensor applications.

\section{Acknowledgements}

We thank the Australian Synchrotron for beam time and the beamline scientists for their help. This research was undertaken in part using the MX2 beamline at the Australian Synchrotron and made use of the ACRF detector. We also thank Alex Caputo for help with the DLS experiments and Lesley Pearce for running the gel-filtration experiment.

\section{References}

Adachi, S. \& Patton, S. (1961). J. Dairy Sci. 44, 1375-1393.

Bern, M., Caval, T., Kil, Y. J., Tang, W., Becker, C., Carlson, E., Kletter, D., Sen, K. I., Galy, N., Hagemans, D., Franc, V. \& Heck, A. J. R. (2018). J. Proteome Res. 17, 1216-1226.

Caron, K. \& Trowell, S. C. (2018). Anal. Chem. 90, 12986-12993.

Emsley, P., Lohkamp, B., Scott, W. G. \& Cowtan, K. (2010). Acta Cryst. D66, 486-501.

Evans, P. R. \& Murshudov, G. N. (2013). Acta Cryst. D69, 1204-1214.

Fazio, V. J., Peat, T. S. \& Newman, J. (2014). Acta Cryst. F70, 13031311.

Hartman, A. H., Liu, H. \& Melville, S. B. (2011). Appl. Environ. Microbiol. 77, 471-478.

Hovey, A. K. \& Frank, D. W. (1995). J. Bacteriol. 177, 4427-4436.

Jain, D. (2015). IUBMB Life, 67, 556-563.

Kabsch, W. (2010). Acta Cryst. D66, 125-132.

Krissinel, E. \& Henrick, K. (2007). J. Mol. Biol. 372, 774-797.

Kwon, H. J., Bennik, M. H. J., Demple, B. \& Ellenberger, T. (2000). Nature Struct. Biol. 7, 424-430.

Laskowski, R. A. \& Swindells, M. B. (2011). J. Chem. Inf. Model. 51, 2778-2786.

Lowden, M. J., Skorupski, K., Pellegrini, M., Chiorazzo, M. G., Taylor, R. K. \& Kull, F. J. (2010). Proc. Natl Acad. Sci. USA, 107, 28602865.

McCoy, A. J., Grosse-Kunstleve, R. W., Adams, P. D., Winn, M. D., Storoni, L. C. \& Read, R. J. (2007). J. Appl. Cryst. 40, 658-674.

Munson, G. P. \& Scott, J. R. (1999). J. Bacteriol. 181, 2110-2117.

Murshudov, G. N., Skubák, P., Lebedev, A. A., Pannu, N. S., Steiner, R. A., Nicholls, R. A., Winn, M. D., Long, F. \& Vagin, A. A. (2011). Acta Cryst. D67, 355-367.

Newman, J., Fazio, V. J., Lawson, B. \& Peat, T. S. (2010). Cryst. Growth Des. 10, 2785-2792.

Rhee, S., Martin, R. G., Rosner, J. L. \& Davies, D. R. (1998). Proc. Natl Acad. Sci. USA, 95, 10413-10418.

Rigali, S., Derouaux, A., Giannotta, F. \& Dusart, J. (2002). J. Biol. Chem. 277, 12507-12515.

Rodgers, M. E. \& Schleif, R. (2009). Proteins, 77, 202-208.

Skubák, P. \& Pannu, N. S. (2013). Nature Commun. 4, 2777.

Soisson, S. M., MacDougall-Shackleton, B., Schleif, R. \& Wolberger, C. (1997). Science, 276, 421-425.

Tobe, T., Schoolnik, G. K., Sohel, I., Bustamante, V. H. \& Puente, J. L. (1996). Mol. Microbiol. 21, 963-975. 